Article

\title{
Sequential Ag-catalyzed carboxylative coupling/Ru-catalyzed cross-metathesis reactions for the synthesis of functionalized 2-alkynoates
}

\author{
ZHANG Linlin, ZHANG Wenzhen*, SHI Linglong, REN Xiang, LÜ Xiaobing\# \\ State Key Laboratory of Fine Chemicals, Dalian University of Technology, Dalian 116024, Liaoning China
}

\section{A R T I C L E I N F O}

Article history:

Received 7 December 2012

Accepted 7 January 2013

Published 20 June 2013

\section{Keywords:}

Carbon dioxide

Carboxylative coupling

Silver catalyst

Cross-metathesis

Grubbs-Hoveyda catalyst

Homogeneous catalysis

\begin{abstract}
A B S T R A C T
Sequential reactions can provide efficient access to a variety of important organic compounds that would be otherwise difficult to obtain using conventional methods and readily available starting materials. Based on the importance of 2-alkynoates in organic synthesis, the current research aimed to develop a method for the convenient synthesis of functionalized 2-alkynoates from terminal alkynes, $\mathrm{CO}_{2}$, terminal alkene-derived bromides, and methyl acrylate using a combination of the carboxylative coupling and cross-metathesis reactions. The initial ligand-free silver-catalyzed carboxylative coupling reactions of a variety of different aryl-substituted terminal alkynes and $\mathrm{CO}_{2}$ with 5-bromopentene and 6-bromohexene provided a series of 4-pentenyl 2-alkynoates and 5-hexenyl 2-alkynoates, respectively, in good yield. These resulting 2-alkynoates were further transformed into methyl (E)-6-acetylenecarboxy-2-hexenoates and (E)-7-acetylenecarboxy-2-heptenoates in moderate to good yields by their cross-metathesis reactions with methyl acrylate in the presence of the Grubbs-Hoveyda catalyst. All of the new products characterized spectroscopically.
\end{abstract}

(C) 2013, Dalian Institute of Chemical Physics, Chinese Academy of Sciences. Published by Elsevier B.V. All rights reserved.

\section{Introduction}

Methods for the synthesis of high value-added fine chemicals using carbon dioxide as a raw material have been the subject of increasing levels of attention during the course of the last two decades, because carbon dioxide is an abundant, inexpensive, and nontoxic renewable $\mathrm{C}_{1}$ feedstock [1-7]. In the presence of suitable homogeneous transition-metal catalysts, carbon dioxide can be efficiently transformed into a variety of different functionalized carboxylic acids and related derivatives [8-22]. The copper- and silver-catalyzed direct carboxylation reactions of terminal alkynes using $\mathrm{CO}_{2}$ as the carboxylative agent have recently been established as efficient and straight- forward methods for the synthesis of functionalized propiolic acids and 2-alkynoates [23,34]. In 2010, we reported the highly selective synthesis of allylic 2-alkynoates according to the carboxylative coupling reaction of terminal alkynes, $\mathrm{CO}_{2}$, and allylic chlorides using a $10 \mathrm{~mol} \%$ loading of (IPr)CuCl (IPr = 1,3-bis(2,6-diisopropylphenyl)imidazol-2-ylidene) as the catalyst [28]. In a later publication, we also disclosed a convenient method for the selective synthesis of functionalized propiolic acids via the direct carboxylation of terminal alkynes with $\mathrm{CO}_{2}$ under ligand-free conditions using only a $1 \mathrm{~mol} \%$ loading of $\mathrm{AgI}$ as the catalyst [30]. More recently, this simple silver-based catalytic system has also been used as a catalyst for the highly efficient and selective carboxylative coupling reaction of a vari-

\footnotetext{
*Corresponding author. Tel: +86-411-84986257; Fax: +86-411-84986256; E-mail: zhangwz@dlut.edu.cn

\#Corresponding author. Tel: +86-411-84986255; Fax: +86-411-84986256; E-mail: lxb-1999@163.com

This work was supported by the National Natural Science Foundation of China (20802007, 21111120073), the Fundamental Research Funds for the Central Universities (DUT12LK47), and the Scientific Research Fund of Liaoning Provincial Education Department (L2012024).

DOI: 10.1016/S1872-2067(12)60527-0 | http://www.sciencedirect.com/science/journal/18722067 | Chin. J. Catal., Vol. 34, No. 6, June 2013
} 
ety of different terminal alkynes, $\mathrm{CO}_{2}$, and chloride compounds to yield 2-alkynoates exclusively. Furthermore, the ligand-free silver catalyst system showed significantly enhanced levels of activity and selectivity at much lower catalyst loadings than the corresponding copper catalyst system [34].

Over the past two decades, olefin metathesis has become one of the most powerful and broadly applicable tools for the construction of carbon-carbon double bonds in organic synthesis [35-39]. In contrast, the cross-metathesis reaction has not been developed to the same extent because of the lack of efficient catalysts capable of controlling the chemoselectivity and stereoselectivity of the reaction [40-44]. Interestingly, however, as a convenient method to gain functionalized and higher olefins from simple alkene precursors, cross-metathesis has emerged as a powerful synthetic strategy and played a crucial role in the total syntheses of many natural products and biologically active compounds, with the utility of technique being significantly enhanced by the development of highly active and selective catalysts [45-50].

Based on the importance of 2-alkynoates in organic synthesis [51-55] and the success of olefin metathesis in a recent study [56], our attentions became focused on the development of a sequential carboxylative coupling/cross-metathesis reaction sequence for the synthesis of functionalized 2-alkynoates from $\mathrm{CO}_{2}$, and other simple and readily available starting materials. With this goal in mind, it was envisaged that the carboxylative coupling of terminal alkynes, $\mathrm{CO}_{2}$, and terminal alkene-derived halides would provide access to terminal alkene-derived 2-alkynoates that could then be subjected to the conditions of the cross-metathesis reactions with an olefin of lower reactivity to give functionalized 2-alkynoates, which would be otherwise difficult to obtain using conventional methods (Scheme 1). Herein, we present an efficient and convenient synthetic protocol for the selective synthesis of functionalized 2-alkynoates in good yields from terminal alkynes, $\mathrm{CO}_{2}$, terminal alkene-derived bromides, and methyl acrylate. The two-steps of this synthetic procedure involved the silver-catalyzed carboxylative coupling and ruthenium-catalyzed cross-metathesis reactions.

\section{Experimental}

Unless otherwise statement, all manipulations were performed using standard Schlenk techniques under a dry argon or $\mathrm{CO}_{2}$ atmosphere. $\mathrm{N}, \mathrm{N}$-Dimethylformamide (DMF) was dis- tilled from $\mathrm{CaH}_{2}$ at $60{ }^{\circ} \mathrm{C}$ under reduced pressure and stored over $4 \mathrm{~A}$ molecular sieves. NMR spectra ( ${ }^{1} \mathrm{H} \mathrm{NMR}, 400 \mathrm{MHz} ;{ }^{13} \mathrm{C}$ NMR, $100 \mathrm{MHz}$ ) were recorded on a Bruker AvanceII 400M type spectrometer (Bruker). Multiplicity abbreviations: $\mathrm{s}=$ singlet, $\mathrm{d}=$ doublet, $\mathrm{t}=$ triplet, $\mathrm{q}=$ quartet, $\mathrm{m}=$ multiplet. High resolution mass spectra (HRMS) were recorded on a Q-TOF mass spectrometer (Micromass, Wythenshawe, UK) equipped with Z-spray ionization source. Infrared (IR) spectra were measured using a Nicolet NEXUS FT-IR spectrophotometer. The second-generation Grubbs catalyst 5 [57,58] and Grubbs-Hoveyda catalyst 6 [59] were synthesized according to the reported procedures. Carbon dioxide (99.999\%), commercially available terminal alkynes, bromide compounds, silver(I) salt, methyl acrylate, and other all of the other reagents were used without further purification.

\subsection{General procedure for the Ag-catalyzed carboxylative coupling of terminal alkynes, $\mathrm{CO}_{2}$, and bromides}

A $70 \mathrm{ml}$ oven dried autoclave containing a stirrer bar was charged the AgI (0.5 mg, $0.002 \mathrm{mmol}$ ) and $\mathrm{Cs}_{2} \mathrm{CO}_{3}$ (978 mg, 3.0 mmol). The autoclave was purged three time with $\mathrm{CO}_{2}$ and the terminal alkynes 1 (2.0 mmol), bromides 2 (3.0 mmol), and dry DMF (20 ml) were then added sequentially via a syringe. The autoclave was then sealed and pressurized to the appropriate pressure with $\mathrm{CO}_{2}$. The reaction mixture was then stirred at 60 ${ }^{\circ} \mathrm{C}$ for $24 \mathrm{~h}$ and the autoclave was cooled to room temperature and the remaining $\mathrm{CO}_{2}$ slowly vented from the system. The reaction mixture was diluted with water $(30 \mathrm{ml})$ and extracted with ethyl acetate $(30 \mathrm{ml} \times 3)$. The combined organic layers were washed with water and brine, dried over $\mathrm{Na}_{2} \mathrm{SO}_{4}$, and filtered. The solvent was removed under vacuum. The product was isolated by column chromatography on silica gel.

All new products prepared by the above procedure were characterized spectroscopically as shown below.

4-Pentenyl phenylpropiolate (3a). 69\% isolated yield. Oil. ${ }^{1} \mathrm{H}$ NMR (400 MHz, $\mathrm{CDCl}_{3}$ ): $\delta 7.61-7.36(\mathrm{~m}, 5 \mathrm{H}, 5 \mathrm{CH}$ ), 5.82 (ddt, $\left.J=16.9,10.2,6.7 \mathrm{~Hz}, 1 \mathrm{H}, \mathrm{CH}=\mathrm{CH}_{2}\right), 5.08(\mathrm{~d}, J=16.9 \mathrm{~Hz}, 1 \mathrm{H}$, trans $\left.\mathrm{CH}=\mathrm{CH}_{2}\right), 5.02\left(\mathrm{~d}, J=10.2 \mathrm{~Hz}, 1 \mathrm{H}\right.$, cis $\left.\mathrm{CH}=\mathrm{CH}_{2}\right), 4.25(\mathrm{t}, J=6.7 \mathrm{~Hz}$, $\left.2 \mathrm{H}, \mathrm{OCH}_{2}\right), 2.21-2.16\left(\mathrm{~m}, 2 \mathrm{H}, \mathrm{CHCH}_{2}\right), 1.86-1.79(\mathrm{~m}, 2 \mathrm{H}$, $\mathrm{CH}_{2} \mathrm{CH}_{2} \mathrm{CH}_{2}$ ). ${ }^{13} \mathrm{C}$ NMR (100 MHz, $\left.\mathrm{CDCl}_{3}\right): \delta 154.10(\mathrm{C}-\mathrm{O}), 137.16$ (vinyl C), 132.97 (aromatic $C$ ), 130.62 (aromatic $C$ ), 128.57 (aromatic $C$ ), 119.64 (aromatic $C$ ), 115.56 (vinyl $C$ ), 86.16 (alkynyl $C$ ), 80.68 (alkynyl $C$ ), $65.42\left(\mathrm{OCH}_{2}\right), 29.89\left(\mathrm{CH}_{2}\right), 27.60$ $\left(\mathrm{CH}_{2}\right)$. IR (neat, $\left.\mathrm{cm}^{-1}\right): 2959,2223,1709,1640,1490,1285$,

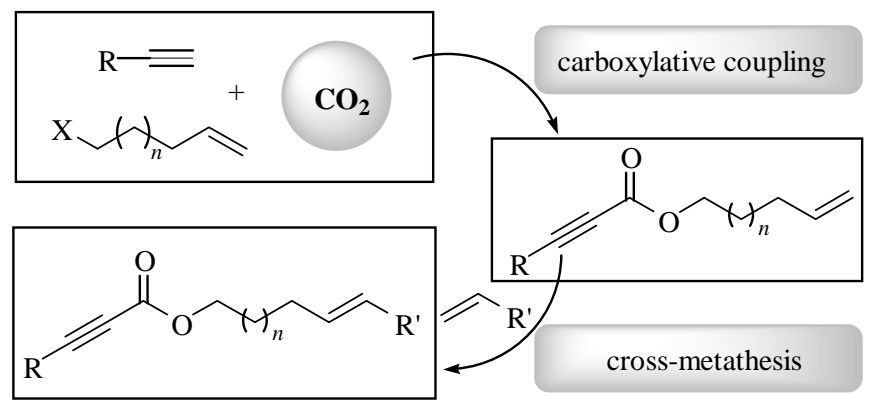

Scheme 1. Synthesis of functionalized 2-alkynoates via the sequential carboxylative coupling/cross-metathesis reactions. 
758, 689. HRMS (ESI, $m / z$ ) calcd. for $\mathrm{C}_{14} \mathrm{H}_{14} \mathrm{O}_{2} \mathrm{Na}[\mathrm{M}+\mathrm{Na}]^{+}$: 237.0891; found: 237.0892 .

4-Pentenyl 4-methylphenylpropiolate (3b). 87\% isolated yield. Oil. ${ }^{1} \mathrm{H}$ NMR (400 MHz, $\left.\mathrm{CDCl}_{3}\right): \delta 7.49(\mathrm{~d}, J=8.0 \mathrm{~Hz}, 2 \mathrm{H}$, $2 \mathrm{CH}$ ), $7.18(\mathrm{~d}, J=8.0 \mathrm{~Hz}, 2 \mathrm{H}, 2 \mathrm{CH}$ ), $5.82(\mathrm{ddt}, J=16.9,10.2,6.7$ $\mathrm{Hz}, 1 \mathrm{H}, \mathrm{CH}=\mathrm{CH}_{2}$ ), 5.07 (d, $J=16.9 \mathrm{~Hz}, 1 \mathrm{H}$, trans $\mathrm{CH}=\mathrm{CH}_{2}$ ), 5.02 (d, $J=10.2 \mathrm{~Hz}, 1 \mathrm{H}$, cis $\left.\mathrm{CH}=\mathrm{CH}_{2}\right), 4.24\left(\mathrm{t}, J=6.7 \mathrm{~Hz}, 2 \mathrm{H}, \mathrm{OCH}_{2}\right)$, 2.38 (s, 2H, CH3), 2.21-2.16 (m, 2H, $\left.\mathrm{CHCH}_{2}\right), 1.86-1.79(\mathrm{~m}, 2 \mathrm{H}$, $\mathrm{CH}_{2} \mathrm{CH}_{2} \mathrm{CH}_{2}$ ). ${ }^{13} \mathrm{C}$ NMR (100 MHz, $\left.\mathrm{CDCl}_{3}\right): \delta 154.25(\mathrm{C}-\mathrm{O}), 141.27$ (aromatic C), 137.19 (vinyl C), 132.98 (aromatic $C$ ), 129.36 (aromatic $C$ ), 116.51 (aromatic $C$ ), 115.53 (vinyl $C$ ), 86.72 (alkynyl $C$ ), 80.33 (alkynyl $C$ ), $65.35\left(\mathrm{OCH}_{2}\right), 29.90\left(\mathrm{CH}_{2}\right), 27.62$ $\left(\mathrm{CH}_{2}\right), 21.69\left(\mathrm{CH}_{3}\right)$. IR (neat, $\left.\mathrm{cm}^{-1}\right): 2957,2217,1708,1641$ 1508, 1290, 817, 748. HRMS (ESI, $m / z$ ) calcd. for $\mathrm{C}_{15} \mathrm{H}_{16} \mathrm{O}_{2} \mathrm{Na}$ [M+Na]+: 251.1048; found: 251.1046.

4-Pentenyl 4-methoxylphenylpropiolate (3c). 91\% isolated yield. Oil. ${ }^{1} \mathrm{H}$ NMR (400 MHz, $\left.\mathrm{CDCl}_{3}\right): \delta 7.54(\mathrm{~d}, J=8.7 \mathrm{~Hz}, 2 \mathrm{H}$, 2CH), $6.88(\mathrm{~d}, J=8.7 \mathrm{~Hz}, 2 \mathrm{H}, 2 \mathrm{CH}$ ), $5.82(\mathrm{ddt}, J=16.9,10.2,6.7$ $\mathrm{Hz}, 1 \mathrm{H}, \mathrm{CH}=\mathrm{CH}_{2}$ ), 5.07 (d, $J=16.9 \mathrm{~Hz}, 1 \mathrm{H}$, trans $\mathrm{CH}=\mathrm{CH}_{2}$ ), 5.02 (d, $J=10.2 \mathrm{~Hz}, 1 \mathrm{H}$, cis $\left.\mathrm{CH}=\mathrm{CH}_{2}\right), 4.23\left(\mathrm{t}, J=6.7 \mathrm{~Hz}, 2 \mathrm{H}, \mathrm{OCH}_{2}\right)$, 3.82 (s, 2H, CH3), 2.20-2.15 (m, 2H, $\left.\mathrm{CHCH}_{2}\right), 1.85-1.78(\mathrm{~m}, 2 \mathrm{H}$, $\mathrm{CH}_{2} \mathrm{CH}_{2} \mathrm{CH}_{2}$ ). ${ }^{13} \mathrm{C}$ NMR (100 MHz, $\mathrm{CDCl}_{3}$ ): $\delta 161.50$ (aromatic $C$ ), 154.37 (C-0), 137.22 (vinyl $C$ ), 134.92 (aromatic $C$ ), 115.52 (vinyl $C$ ), 114.28 (aromatic $C$ ), 111.36 (aromatic $C$ ), 87.02 (alkynyl $C$ ), 80.10 (alkynyl $C$ ), $65.27\left(\mathrm{OCH}_{2}\right), 55.37\left(\mathrm{OCH}_{3}\right), 29.91$ $\left(\mathrm{CH}_{2}\right), 27.62\left(\mathrm{CH}_{2}\right)$. IR (neat, $\left.\mathrm{cm}^{-1}\right): 2959,2210,1705,1605$ $1510,1286,834,747$. HRMS (ESI, $m / z$ ) calcd. for $\mathrm{C}_{15} \mathrm{H}_{16} \mathrm{O}_{3} \mathrm{Na}$ [M+Na] ${ }^{+}: 267.0997$; found: 267.0993.

4-Pentenyl 4-tert-butylphenylpropiolate (3d). 87\% isolated yield. Oil. ${ }^{1} \mathrm{H}$ NMR (400 MHz, $\left.\mathrm{CDCl}_{3}\right): \delta 7.54(\mathrm{~d}, J=8.2 \mathrm{~Hz}, 2 \mathrm{H}$, $2 \mathrm{CH}$ ), $7.40(\mathrm{~d}, J=8.2 \mathrm{~Hz}, 2 \mathrm{H}, 2 \mathrm{CH}$ ), $5.82(\mathrm{ddt}, J=16.9,10.2,6.7$ $\mathrm{Hz}, 1 \mathrm{H}, \mathrm{CH}=\mathrm{CH}_{2}$ ), 5.08 (d, $J=16.9 \mathrm{~Hz}, 1 \mathrm{H}$, trans $\left.\mathrm{CH}=\mathrm{CH}_{2}\right), 5.02$ $\left(\mathrm{d}, J=10.2 \mathrm{~Hz}, 1 \mathrm{H}\right.$, cis $\left.\mathrm{CH}=\mathrm{CH}_{2}\right), 4.25\left(\mathrm{t}, J=6.7 \mathrm{~Hz}, 2 \mathrm{H}, \mathrm{OCH}_{2}\right)$ 2.21-2.16 (m, 2H, $\mathrm{CHCH}_{2}$ ), 1.85-1.79 (m, 2H, $\mathrm{CH}_{2} \mathrm{CH}_{2} \mathrm{CH}_{2}$ ), 1.32 $\left(\mathrm{s}, 9 \mathrm{H}, \mathrm{CH}_{3}\right) .{ }^{13} \mathrm{C}$ NMR $\left(100 \mathrm{MHz}, \mathrm{CDCl}_{3}\right): \delta 154.26$ (aromatic $C$ ), 154.23 (C-0), 137.18 (vinyl $C$ ), 132.89 (aromatic $C$ ), 125.64 (aromatic $C$ ), 116.53 (aromatic $C$ ), 115.55 (vinyl $C$ ), 86.65 (alkynyl $C$ ), 80.35 (alkynyl $C$ ), $65.32\left(\mathrm{OCH}_{2}\right), 35.01\left(\mathrm{C}\left(\mathrm{CH}_{3}\right)_{3}\right)$, $31.04\left(\mathrm{CH}_{3}\right), 29.92\left(\mathrm{CH}_{2}\right), 27.61\left(\mathrm{CH}_{2}\right)$. IR (neat, $\left.\mathrm{cm}^{-1}\right): 2964$, 2220, 1709, 1290, 1200, 1117, 837, 748. HRMS (ESI, $m / z$ ) calcd. for $\mathrm{C}_{18} \mathrm{H}_{22} \mathrm{O}_{2} \mathrm{Na}[\mathrm{M}+\mathrm{Na}]^{+}$: 293.1517; found: 293.1519 .

4-Pentenyl 4-trifluoromethylphenylpropiolate (3e). 85\%

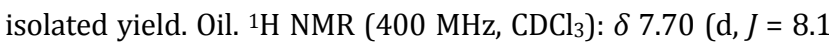
$\mathrm{Hz}, 2 \mathrm{H}, 2 \mathrm{CH}$ ), $7.64(\mathrm{~d}, J=8.1 \mathrm{~Hz}, 2 \mathrm{H}, 2 \mathrm{CH}$ ), 5.82 (ddt, $J=16.9$, 10.2, $\left.6.7 \mathrm{~Hz}, 1 \mathrm{H}, \mathrm{CH}=\mathrm{CH}_{2}\right), 5.08(\mathrm{~d}, J=16.9 \mathrm{~Hz}, 1 \mathrm{H}$, trans $\left.\mathrm{CH}=\mathrm{CH}_{2}\right), 5.02\left(\mathrm{~d}, J=10.2 \mathrm{~Hz}, 1 \mathrm{H}\right.$, cis $\left.\mathrm{CH}=\mathrm{CH}_{2}\right), 4.27(\mathrm{t}, J=6.7 \mathrm{~Hz}$ $\left.2 \mathrm{H}, \mathrm{OCH}_{2}\right), 2.21-2.16\left(\mathrm{~m}, 2 \mathrm{H}, \mathrm{CHCH}_{2}\right), 1.87-1.80(\mathrm{~m}, 2 \mathrm{H}$ $\mathrm{CH}_{2} \mathrm{CH}_{2} \mathrm{CH}_{2}$ ). ${ }^{13} \mathrm{C}$ NMR (100 MHz, $\left.\mathrm{CDCl}_{3}\right): \delta 153.78(\mathrm{C}-\mathrm{O}), 137.19$ (vinyl C), 133.29 (aromatic C), 132.49 (aromatic C), 132.14 (aromatic $C$ ), 125.67 (aromatic $C$ ), $123.63\left(\mathrm{CF}_{3}\right), 115.78$ (vinyl C), 84.06 (alkynyl C), 82.40 (alkynyl C), $65.87\left(\mathrm{OCH}_{2}\right), 30.01$ $\left(\mathrm{CH}_{2}\right), 27.70\left(\mathrm{CH}_{2}\right)$. IR (neat, $\left.\mathrm{cm}^{-1}\right): 2960,2232,1715,1324$ 1288, 1197, 1178, 1132, 1067, 844, 748. HRMS (ESI, $\mathrm{m} / z$ ) calcd. for $\mathrm{C}_{15} \mathrm{H}_{13} \mathrm{O}_{2} \mathrm{~F}_{3} \mathrm{Na}[\mathrm{M}+\mathrm{Na}]^{+}: 305.0765$; found: 305.0774 .

4-Pentenyl 5-phenyl-2-pentynoate (3f). 65\% isolated yield. Oil. ${ }^{1} \mathrm{H}$ NMR (400 MHz, $\mathrm{CDCl}_{3}$ ): $\delta 7.33-7.21(\mathrm{~m}, 5 \mathrm{H}, 5 \mathrm{CH}), 5.80$ (ddt, $J=16.9,10.2,6.7 \mathrm{~Hz}, 1 \mathrm{H}, \mathrm{CH}=\mathrm{CH}_{2}$ ), 5.05 (d, $J=16.9 \mathrm{~Hz}, 1 \mathrm{H}$, trans $\left.\mathrm{CH}=\mathrm{CH}_{2}\right), 5.00\left(\mathrm{~d}, J=10.2 \mathrm{~Hz}, 1 \mathrm{H}\right.$, cis $\left.\mathrm{CH}=\mathrm{CH}_{2}\right), 4.16(\mathrm{t}, J=$ $\left.6.7 \mathrm{~Hz}, 2 \mathrm{H}, \mathrm{OCH}_{2}\right), 2.90\left(\mathrm{t}, J=7.6 \mathrm{~Hz}, 2 \mathrm{H}, \mathrm{CH}_{2}\right), 2.62(\mathrm{t}, J=7.6 \mathrm{~Hz}$, $\left.2 \mathrm{H}, \mathrm{CH}_{2}\right), 2.17-2.12\left(\mathrm{~m}, 2 \mathrm{H}, \mathrm{CHCH}_{2}\right), 1.81-1.74(\mathrm{~m}, 2 \mathrm{H}$, $\mathrm{CH}_{2} \mathrm{CH}_{2} \mathrm{CH}_{2}$ ). ${ }^{13} \mathrm{C}$ NMR (100 MHz, $\mathrm{CDCl}_{3}$ ): $\delta 153.76(C-0), 139.63$ (aromatic C), 137.21 (vinyl $C$ ), 128.59 (aromatic $C$ ), 128.38 (aromatic $C$ ), 126.67 (aromatic $C$ ), 115.53 (vinyl $C$ ), 88.40 (alkynyl $C$ ), 73.74 (alkynyl $C$ ), $65.16\left(\mathrm{OCH}_{2}\right), 33.85\left(\mathrm{CH}_{2}\right), 29.91$ $\left(\mathrm{CH}_{2}\right), 27.56\left(\mathrm{CH}_{2}\right), 20.88\left(\mathrm{CH}_{2}\right)$. IR (neat, $\left.\mathrm{cm}^{-1}\right): 2954,2237$, $1709,1258,1070,750,699$. HRMS (ESI, $m / z$ ) calcd. for $\mathrm{C}_{16} \mathrm{H}_{18} \mathrm{O}_{2} \mathrm{Na}[\mathrm{M}+\mathrm{Na}]^{+}: 265.1204$; found: 265.1211 .

4-Pentenyl 2-nonynoate (3g). 64\% isolated yield. Oil. ${ }^{1} \mathrm{H}$ NMR (400 MHz, $\mathrm{CDCl}_{3}$ ): $\delta 5.80(\mathrm{ddt}, J=16.9,10.2,6.7 \mathrm{~Hz}, 1 \mathrm{H}$, $\left.\mathrm{C} H=\mathrm{CH}_{2}\right), 5.04\left(\mathrm{~d}, J=16.9 \mathrm{~Hz}, 1 \mathrm{H}\right.$, trans $\left.\mathrm{CH}=\mathrm{CH}_{2}\right), 5.00(\mathrm{~d}, J=$ $10.2 \mathrm{~Hz}, 1 \mathrm{H}$, cis $\left.\mathrm{CH}=\mathrm{CH}_{2}\right), 4.17\left(\mathrm{t}, J=6.7 \mathrm{~Hz}, 2 \mathrm{H}, \mathrm{OCH}_{2}\right), 2.33(\mathrm{t}, J$ $\left.=7.2 \mathrm{~Hz}, 2 \mathrm{H}, \mathrm{CH}_{2}\right), 2.17-2.12\left(\mathrm{~m}, 2 \mathrm{H}, \mathrm{CHCH}_{2}\right), 1.81-1.74(\mathrm{~m}, 2 \mathrm{H}$, $\mathrm{CH}_{2} \mathrm{CH}_{2} \mathrm{CH}_{2}$ ), $1.61-1.29$ (m, 8H, 4CH $), 0.89$ (t, $J=6.5 \mathrm{~Hz}, 3 \mathrm{H}$, $\mathrm{CH}_{3}$ ). ${ }^{13} \mathrm{C}$ NMR (100 MHz, $\mathrm{CDCl}_{3}$ ): $\delta 153.93$ (C-0), 137.17 (vinyl $C$ ), 115.43 (vinyl $C$ ), 89.60 (alkynyl $C$ ), 73.07 (alkynyl $C$ ), 65.09 $\left(\mathrm{OCH}_{2}\right), 31.17\left(\mathrm{CH}_{2}\right), 29.86\left(\mathrm{CH}_{2}\right), 28.48\left(\mathrm{CH}_{2}\right), 27.53\left(\mathrm{CH}_{2}\right)$, $27.47\left(\mathrm{CH}_{2}\right), 22.42\left(\mathrm{CH}_{2}\right), 18.65\left(\mathrm{CH}_{2}\right), 13.96\left(\mathrm{CH}_{3}\right)$. IR (neat, $\mathrm{cm}^{-1}$ ): 2959, 2236, 1713, 1642, 1258, 1083. HRMS (ESI, $\mathrm{m} / \mathrm{z}$ ) calcd. for $\mathrm{C}_{14} \mathrm{H}_{22} \mathrm{O}_{2} \mathrm{Na}$ [M+Na]+: 245.1517; found: 245.1521 .

5-Hexenyl phenylpropiolate (4a). $75 \%$ isolated yield. Oil. ${ }^{1} \mathrm{H}$ NMR (400 MHz, $\mathrm{CDCl}_{3}$ ): $\delta 7.60-7.36(\mathrm{~m}, 5 \mathrm{H}, 5 \mathrm{CH}$ ), 5.80 (ddt, $J=$ $\left.16.9,10.2,6.7 \mathrm{~Hz}, 1 \mathrm{H}, \mathrm{CH}=\mathrm{CH}_{2}\right), 5.04(\mathrm{~d}, J=16.9 \mathrm{~Hz}, 1 \mathrm{H}$, trans $\left.\mathrm{CH}=\mathrm{CH}_{2}\right), 4.98\left(\mathrm{~d}, J=10.2 \mathrm{~Hz}, 1 \mathrm{H}\right.$, cis $\left.\mathrm{CH}=\mathrm{CH}_{2}\right), 4.25(\mathrm{t}, J=6.7 \mathrm{~Hz}$, $\left.2 \mathrm{H}, \mathrm{OCH}_{2}\right), 2.14-2.11\left(\mathrm{~m}, 2 \mathrm{H}, \mathrm{CHCH}_{2}\right), 1.76-1.72(\mathrm{~m}, 2 \mathrm{H}$, $\left.\mathrm{CH}_{2} \mathrm{CH}_{2} \mathrm{CH}_{2}\right)$, $1.54-1.50\left(\mathrm{~m}, 2 \mathrm{H}, \mathrm{CH}_{2}\right) .{ }^{13} \mathrm{C} \mathrm{NMR}(100 \mathrm{MHz}$, $\mathrm{CDCl}_{3}$ ): $\delta 154.17$ (C-0), 138.19 (vinyl $C$ ), 132.98 (aromatic $C$ ), 130.63 (aromatic $C$ ), 128.58 (aromatic $C$ ), 119.63 (aromatic $C$ ), 115.00 (vinyl $C$ ), 86.12 (alkynyl $C$ ), 80.70 (alkynyl $C$ ), 65.99 $\left(\mathrm{OCH}_{2}\right), 33.24\left(\mathrm{CH}_{2}\right), 27.88\left(\mathrm{CH}_{2}\right), 25.07\left(\mathrm{CH}_{2}\right)$. IR (neat, $\left.\mathrm{cm}^{-1}\right)$ : 2936, 2221, 1709, 1640, 1490, 1286, 758, 689. HRMS (ESI, $m / z$ ) calcd. for $\mathrm{C}_{15} \mathrm{H}_{16} \mathrm{O}_{2} \mathrm{Na}[\mathrm{M}+\mathrm{Na}]^{+}$: 251.1048; found: 251.1045 .

5-Hexenyl 4-methylphenylpropiolate (4b). 76\% isolated yield. Oil. ${ }^{1} \mathrm{H}$ NMR (400 MHz, $\left.\mathrm{CDCl}_{3}\right): \delta 7.50(\mathrm{~d}, J=8.0 \mathrm{~Hz}, 2 \mathrm{H}$, 2CH), 7.19 (d, $J=8.0 \mathrm{~Hz}, 2 \mathrm{H}, 2 \mathrm{CH}$ ), 5.82 (ddt, $J=16.9,10.2,6.7$ $\mathrm{Hz}, 1 \mathrm{H}, \mathrm{CH}=\mathrm{CH}_{2}$ ), $5.04\left(\mathrm{~d}, J=16.9 \mathrm{~Hz}, 1 \mathrm{H}\right.$, trans $\left.\mathrm{CH}=\mathrm{CH}_{2}\right), 4.99$ (d, $J=10.2 \mathrm{~Hz}, 1 \mathrm{H}$, cis $\mathrm{CH}=\mathrm{CH}_{2}$ ), $4.24\left(\mathrm{t}, J=6.7 \mathrm{~Hz}, 2 \mathrm{H}, \mathrm{OCH}_{2}\right.$ ), 2.39 (s, 2H, $\left.\mathrm{CH}_{3}\right), 2.15-2.09\left(\mathrm{~m}, 2 \mathrm{H}, \mathrm{CHCH}_{2}\right), 1.76-1.71(\mathrm{~m}, 2 \mathrm{H}$, $\left.\mathrm{CH}_{2} \mathrm{CH}_{2} \mathrm{CH}_{2}\right)$, $1.56-1.50\left(\mathrm{~m}, 2 \mathrm{H}, \mathrm{CH}_{2}\right) .{ }^{13} \mathrm{C} \mathrm{NMR}(100 \mathrm{MHz}$, $\mathrm{CDCl}_{3}$ ): $\delta 154.25$ (C-0), 141.23 (aromatic $C$ ), 138.17 (vinyl $C$ ), 132.95 (aromatic $C$ ), 129.35 (aromatic $C$ ), 116.52 (aromatic $C$ ), 114.95 (vinyl $C$ ), 86.62 (alkynyl $C$ ), 80.38 (alkynyl $C$ ), 65.85 $\left(\mathrm{OCH}_{2}\right), 33.23\left(\mathrm{CH}_{2}\right), 27.90\left(\mathrm{CH}_{2}\right), 25.08\left(\mathrm{CH}_{2}\right), 21.66\left(\mathrm{CH}_{3}\right) . \mathrm{IR}$ (neat, $\mathrm{cm}^{-1}$ ): 2937, 2218, 1709, 1641, 1509, 1290, 817, 748. HRMS (ESI, $m / z$ ) calcd. for $\mathrm{C}_{16} \mathrm{H}_{18} \mathrm{O}_{2} \mathrm{Na}[\mathrm{M}+\mathrm{Na}]^{+}:$265.1204; found: 265.1211 .

5-Hexenyl 4-methoxylphenylpropiolate (4c). 86\% isolated yield. Oil. ${ }^{1} \mathrm{H}$ NMR (400 MHz, $\left.\mathrm{CDCl}_{3}\right): \delta 7.45(\mathrm{~d}, J=8.7 \mathrm{~Hz}, 2 \mathrm{H}$, 2CH), 6.79 (d, $J=8.7 \mathrm{~Hz}, 2 \mathrm{H}, 2 \mathrm{CH}$ ), 5.72 (ddt, $J=16.9,10.2,6.7$ $\mathrm{Hz}, 1 \mathrm{H}, \mathrm{CH}=\mathrm{CH}_{2}$ ), 4.95 (d, $J=16.9 \mathrm{~Hz}, 1 \mathrm{H}$, trans $\mathrm{CH}=\mathrm{CH}_{2}$ ), 4.89 (d, $J=10.2 \mathrm{~Hz}, 1 \mathrm{H}$, cis $\mathrm{CH}=\mathrm{CH}$ ), $4.14\left(\mathrm{t}, J=6.7 \mathrm{~Hz}, 2 \mathrm{H}, \mathrm{OCH}_{2}\right.$ ), $3.74\left(\mathrm{~s}, 2 \mathrm{H}, \mathrm{CH}_{3}\right), 2.05-1.99\left(\mathrm{~m}, 2 \mathrm{H}, \mathrm{CHCH}_{2}\right), 1.67-1.60(\mathrm{~m}, 2 \mathrm{H}$, $\left.\mathrm{CH}_{2} \mathrm{CH}_{2} \mathrm{CH}_{2}\right), 1.46-1.38\left(\mathrm{~m}, 2 \mathrm{H}, \mathrm{CH}_{2}\right) .{ }^{13} \mathrm{C} \mathrm{NMR}(100 \mathrm{MHz}$, $\mathrm{CDCl}_{3}$ ): $\delta 161.49$ (aromatic $C$ ), $154.39(C-0), 138.20$ (vinyl $C$ ), 
134.90 (aromatic $C$ ), 114.94 (vinyl $C$ ), 114.27 (aromatic $C$ ), 111.37 (aromatic $C$ ), 86.94 (alkynyl $C$ ), 80.14 (alkynyl $C$ ), 65.78 $\left(\mathrm{OCH}_{2}\right), 55.35\left(\mathrm{OCH}_{3}\right), 33.23\left(\mathrm{CH}_{2}\right), 27.91\left(\mathrm{CH}_{2}\right), 25.08\left(\mathrm{CH}_{2}\right)$. IR (neat, $\mathrm{cm}^{-1}$ ): 2937, 2214, 1705, 1604, 1510, 1288, 834, 747. HRMS (ESI, $m / z$ ) calcd. for $\mathrm{C}_{16} \mathrm{H}_{18} \mathrm{O}_{3} \mathrm{Na}[\mathrm{M}+\mathrm{Na}]^{+}$: 281.1154; found: 281.1162 .

5-Hexenyl 4-tert-butylphenylpropiolate (4d). $88 \%$ isolated yield. Oil. ${ }^{1} \mathrm{H}$ NMR ( $400 \mathrm{MHz}, \mathrm{CDCl}_{3}$ ): $\delta 7.53$ (d, $J=8.5 \mathrm{~Hz}, 2 \mathrm{H}$, $2 \mathrm{CH}$ ), $7.39(\mathrm{~d}, J=8.5 \mathrm{~Hz}, 2 \mathrm{H}, 2 \mathrm{CH}$ ), 5.81 (ddt, $J=16.9,10.2,6.7$ $\left.\mathrm{Hz}, 1 \mathrm{H}, \mathrm{CH}=\mathrm{CH}_{2}\right), 5.04\left(\mathrm{~d}, J=16.9 \mathrm{~Hz}, 1 \mathrm{H}\right.$, trans $\left.\mathrm{CH}=\mathrm{CH}_{2}\right), 4.98$ (d, $J=10.2 \mathrm{~Hz}, 1 \mathrm{H}$, cis $\left.\mathrm{CH}=\mathrm{CH}_{2}\right), 4.24\left(\mathrm{t}, J=6.6 \mathrm{~Hz}, 2 \mathrm{H}, \mathrm{OCH}_{2}\right)$, 2.14-2.09 (m, $\left.2 \mathrm{H}, \mathrm{CHCH}_{2}\right), 1.77-1.70\left(\mathrm{~m}, 2 \mathrm{H}, \mathrm{CH}_{2} \mathrm{CH}_{2} \mathrm{CH}_{2}\right)$, 1.56-1.48 (m, 2H, $\left.\mathrm{CH}_{2}\right), 1.32$ (s, 9H, $\left.\mathrm{CH}_{3}\right) .{ }^{13} \mathrm{C}$ NMR $(100 \mathrm{MHz}$, $\mathrm{CDCl}_{3}$ ): $\delta 154.18$ (aromatic $C$ ), 154.16 (C-0), 138.11 (vinyl $C$ ), 132.85 (aromatic $C$ ), 125.60 (aromatic $C$ ), 116.58 (aromatic $C$ ), 114.97 (vinyl $C$ ), 86.49 (alkynyl $C$ ), 80.43 (alkynyl $C$ ), 65.78 $\left(\mathrm{OCH}_{2}\right), 34.96\left(\mathrm{C}\left(\mathrm{CH}_{3}\right)_{3}\right), 33.24\left(\mathrm{CH}_{3}\right), 31.01\left(\mathrm{CH}_{2}\right), 27.91\left(\mathrm{CH}_{2}\right)$, $25.08\left(\mathrm{CH}_{2}\right)$. IR (neat, $\mathrm{cm}^{-1}$ ): 2964, 2220, 1709, 1290, 1201, 1175, 837, 748. HRMS (ESI, $\mathrm{m} / z$ ) calcd. for $\mathrm{C}_{19} \mathrm{H}_{24} \mathrm{O}_{2} \mathrm{Na}$ [M+Na]+: 307.1674; found: 307.1678 .

5-Hexenyl 4-trifluoromethylphenylpropiolate (4e). 83\% isolated yield. Oil. ${ }^{1} \mathrm{H}$ NMR $\left(400 \mathrm{MHz}, \mathrm{CDCl}_{3}\right): \delta 7.70$ (d, $J=8.1$ $\mathrm{Hz}, 2 \mathrm{H}, 2 \mathrm{CH}$ ), $7.64(\mathrm{~d}, J=8.1 \mathrm{~Hz}, 2 \mathrm{H}, 2 \mathrm{CH}$ ), 5.81 (ddt, $J=16.9$, $\left.10.2,6.7 \mathrm{~Hz}, 1 \mathrm{H}, \mathrm{CH}=\mathrm{CH}_{2}\right), 5.03(\mathrm{~d}, J=16.9 \mathrm{~Hz}, 1 \mathrm{H}$, trans $\left.\mathrm{CH}=\mathrm{CH}_{2}\right), 4.99\left(\mathrm{~d}, J=10.2 \mathrm{~Hz}, 1 \mathrm{H}\right.$, cis $\left.\mathrm{CH}=\mathrm{CH}_{2}\right), 4.26(\mathrm{t}, J=6.7 \mathrm{~Hz}$, $\left.2 \mathrm{H}, \mathrm{OCH}_{2}\right), 2.14-2.09\left(\mathrm{~m}, 2 \mathrm{H}, \mathrm{CHCH}_{2}\right), 1.78-1.71(\mathrm{~m}, 2 \mathrm{H}$, $\left.\mathrm{CH}_{2} \mathrm{CH}_{2} \mathrm{CH}_{2}\right), 1.56-1.48\left(\mathrm{~m}, 2 \mathrm{H}, \mathrm{CH}_{2}\right) .{ }^{13} \mathrm{C}$ NMR $(100 \mathrm{MHz}$, $\mathrm{CDCl}_{3}$ ): $\delta 153.68(C-0), 138.11$ (vinyl $C$ ), 133.14 (aromatic $C$ ), 132.33 (aromatic $C$ ), 132.00 (aromatic $C$ ), 125.51 (aromatic $C$ ), $123.50\left(\mathrm{CF}_{3}\right.$ ), 115.02 (vinyl $C$ ), 83.87 (alkynyl $C$ ), 82.29 (alkynyl C), $66.27\left(\mathrm{OCH}_{2}\right), 33.21\left(\mathrm{CH}_{2}\right), 27.85\left(\mathrm{CH}_{2}\right), 25.06\left(\mathrm{CH}_{2}\right)$. IR (neat, $\mathrm{cm}^{-1}$ ): 2938, 2228, 1716, 1324, 1289, 1197, 1177, 1133, 1068, 844, 748. HRMS (ESI, $\mathrm{m} / \mathrm{z}$ ) calcd. for $\mathrm{C}_{16} \mathrm{H}_{15} \mathrm{O}_{2} \mathrm{~F}_{3} \mathrm{Na}$ [M+Na]+: 319.0922; found: 319.0926 .

5-Hexenyl 5-phenyl-2-pentynoate (4f). $66 \%$ isolated yield. Oil. ${ }^{1} \mathrm{H}$ NMR (400 MHz, $\mathrm{CDCl}_{3}$ ): $\delta 7.33-7.21(\mathrm{~m}, 5 \mathrm{H}, 5 \mathrm{CH}$ ), 5.80 (ddt, $J=16.9,10.2,6.7 \mathrm{~Hz}, 1 \mathrm{H}, \mathrm{CH}=\mathrm{CH}_{2}$ ), $5.03(\mathrm{~d}, J=16.9 \mathrm{~Hz}, 1 \mathrm{H}$, trans $\left.\mathrm{CH}=\mathrm{CH}_{2}\right), 4.98\left(\mathrm{~d}, J=10.2 \mathrm{~Hz}, 1 \mathrm{H}\right.$, cis $\left.\mathrm{CH}=\mathrm{CH}_{2}\right), 4.16(\mathrm{t}, J=$ $\left.6.6 \mathrm{~Hz}, 2 \mathrm{H}, \mathrm{OCH}_{2}\right), 2.90\left(\mathrm{t}, J=7.6 \mathrm{~Hz}, 2 \mathrm{H}, \mathrm{CH}_{2}\right), 2.63(\mathrm{t}, J=7.7 \mathrm{~Hz}$, $\left.2 \mathrm{H}, \mathrm{CH}_{2}\right), 2.12-2.06\left(\mathrm{~m}, 2 \mathrm{H}, \mathrm{CHCH}_{2}\right), 1.72-1.65(\mathrm{~m}, 2 \mathrm{H}$, $\left.\mathrm{CH}_{2} \mathrm{CH}_{2} \mathrm{CH}_{2}\right), 1.51-1.44\left(\mathrm{~m}, 2 \mathrm{H}, \mathrm{CH}_{2}\right) .{ }^{13} \mathrm{C}$ NMR $(100 \mathrm{MHz}$, $\mathrm{CDCl}_{3}$ ): $\delta 153.80(C-0), 139.64$ (aromatic $C$ ), 138.21 (vinyl $C$ ), 128.58 (aromatic $C$ ), 128.37 (aromatic $C$ ), 126.66 (aromatic $C$ ), 114.97 (vinyl $C$ ), 88.34 (alkynyl $C$ ), 73.77 (alkynyl $C$ ), 65.71 $\left(\mathrm{OCH}_{2}\right), 33.86\left(\mathrm{CH}_{2}\right), 33.24\left(\mathrm{CH}_{2}\right), 27.85\left(\mathrm{CH}_{2}\right), 25.08\left(\mathrm{CH}_{2}\right)$, $20.88\left(\mathrm{CH}_{2}\right)$. IR (neat, $\left.\mathrm{cm}^{-1}\right): 2934,2236,1709,1252,1070$, 750, 699. HRMS (ESI, $\mathrm{m} / \mathrm{z}$ ) calcd. for $\mathrm{C}_{17} \mathrm{H}_{20} \mathrm{O}_{2} \mathrm{Na}[\mathrm{M}+\mathrm{Na}]^{+}$: 279.1361; found: 279.1372 .

5-Hexenyl 2-nonynoate (4g). 59\% isolated yield. Oil. ${ }^{1} \mathrm{H}$ NMR (400 MHz, CDCl 3 ): $\delta 5.81$ (ddt, $J=16.9,10.2,6.7 \mathrm{~Hz}, 1 \mathrm{H}$, $\left.\mathrm{CH}=\mathrm{CH}_{2}\right), 5.02\left(\mathrm{~d}, J=16.9 \mathrm{~Hz}, 1 \mathrm{H}\right.$, trans $\left.\mathrm{CH}=\mathrm{CH}_{2}\right), 4.97(\mathrm{~d}, J=$ $10.2 \mathrm{~Hz}, 1 \mathrm{H}$, cis $\left.\mathrm{CH}=\mathrm{CH}_{2}\right), 4.16\left(\mathrm{t}, J=6.7 \mathrm{~Hz}, 2 \mathrm{H}, \mathrm{OCH} \mathrm{H}_{2}\right), 2.33(\mathrm{t}, J$ $\left.=7.1 \mathrm{~Hz}, 2 \mathrm{H}, \mathrm{CH}_{2}\right), 2.11-2.06\left(\mathrm{~m}, 2 \mathrm{H}, \mathrm{CHCH}_{2}\right), 1.72-1.30(\mathrm{~m}$, $\left.12 \mathrm{H}, 6 \mathrm{CH}_{2}\right), 0.89\left(\mathrm{t}, J=6.5 \mathrm{~Hz}, 3 \mathrm{H}, \mathrm{CH}_{3}\right) .{ }^{13} \mathrm{C}$ NMR $(100 \mathrm{MHz}$, $\mathrm{CDCl}_{3}$ ): $\delta 153.95(C-0), 138.15$ (vinyl $C$ ), 114.87 (vinyl $C$ ), 89.50 (alkynyl C), 73.09 (alkynyl $\mathrm{C}$ ), $65.61\left(\mathrm{OCH}_{2}\right), 33.20\left(\mathrm{CH}_{2}\right), 31.17$ $\left(\mathrm{CH}_{2}\right), 28.48\left(\mathrm{CH}_{2}\right), 27.80\left(\mathrm{CH}_{2}\right), 27.47\left(\mathrm{CH}_{2}\right), 25.02\left(\mathrm{CH}_{2}\right), 22.42$
$\left(\mathrm{CH}_{2}\right), 18.63\left(\mathrm{CH}_{2}\right), 13.96\left(\mathrm{CH}_{3}\right)$. IR (neat, $\left.\mathrm{cm}^{-1}\right): 2954,2236$, $1713,1641,1250,1083$. HRMS (ESI, $m / z$ ) calcd. for $\mathrm{C}_{15} \mathrm{H}_{24} \mathrm{O}_{2} \mathrm{Na}$ [M+Na]+: 259.1674; found: 259.1683.

\subsection{General procedure for the cross-metathesis reaction of the terminal alkene-derived 2-alkynoates with methyl acrylate}

To a solution of the terminal alkene-derived 2-alkynoates 3 or $4(0.2 \mathrm{mmol})$ in toluene $(0.1 \mathrm{~mol} / \mathrm{L})$ in a Schlenk tube containing a stirrer bar were added methyl acrylates (50 equiv) and the Grubbs-Hoveyda catalyst ( $5 \mathrm{~mol} \%$ ). The resulting reaction mixtures were heated under an argon atmosphere at $60^{\circ} \mathrm{C}$ for $12 \mathrm{~h}$. The mixtures were then quickly filtered through silica and the solvent removed under reduced pressure to give the crude residues, which were purified by flash column chromatography on silica gel to provide the cross-metathesis products 8 or 9.

All new products prepared by the above procedure were characterized spectroscopically as shown below.

Methyl (E)-6-phenylacetylenecarboxy-2-hexenoate (8a). $31 \%$ isolated yield. Oil. ${ }^{1} \mathrm{H}$ NMR ( $400 \mathrm{MHz}, \mathrm{CDCl}_{3}$ ): $\delta 7.60-7.36$ (m, 5H, 5CH), $6.97\left(\mathrm{dt}, J=15.6,6.9 \mathrm{~Hz}, 1 \mathrm{H}, \mathrm{CHCH}_{2}\right), 5.89(\mathrm{~d}, J=$ $15.6,1 \mathrm{H}, \mathrm{CHCO}), 4.26\left(\mathrm{t}, J=6.9 \mathrm{~Hz}, 2 \mathrm{H}, \mathrm{OCH}_{2}\right), 3.73(\mathrm{~s}, 3 \mathrm{H}$, $\left.\mathrm{OCH}_{3}\right), 2.38-2.32$ (m, 2H, $\left.\mathrm{CHCH}_{2}\right), 1.93-1.86 \quad(\mathrm{~m}, 2 \mathrm{H}$, $\mathrm{CH}_{2} \mathrm{CH}_{2} \mathrm{CH}_{2}$ ). ${ }^{13} \mathrm{C}$ NMR (100 MHz, $\left.\mathrm{CDCl}_{3}\right): \delta 166.84(C-0), 154.00$ (C-0), 147.51 (alkenyl C), 133.01 (aromatic C), 130.69 (aromatic $C$ ), 128.58 (aromatic $C$ ), 121.89 (alkenyl $C$ ), 119.55 (aromatic $C$ ), 86.54 (alkynyl $C$ ), 80.47 (alkynyl $C$ ), $64.98\left(\mathrm{OCH}_{2}\right.$ ), $51.47\left(\mathrm{OCH}_{3}\right), 28.52\left(\mathrm{CH}_{2}\right), 26.91\left(\mathrm{CH}_{2}\right)$. IR (neat, $\left.\mathrm{cm}^{-1}\right): 2956$, 2924, 2219, 1716, 1660, 1463, 1278, 1261, 1024, 757, 689. HRMS (ESI, $m / z$ ) calcd. for $\mathrm{C}_{16} \mathrm{H}_{16} \mathrm{O}_{4} \mathrm{Na}[\mathrm{M}+\mathrm{Na}]^{+}:$295.0946; found: 295.0935 .

Methyl (E)-6-(4-methylphenyl)acetylenecarboxy-2-hexenoate (8b). $63 \%$ isolated yield. Oil. ${ }^{1} \mathrm{H}$ NMR $\left(400 \mathrm{MHz}, \mathrm{CDCl}_{3}\right): \delta$ 7.49 (d, $J=8.0 \mathrm{~Hz}, 2 \mathrm{H}, 2 \mathrm{CH}$ ), 7.19 (d, $J=8.0 \mathrm{~Hz}, 2 \mathrm{H}, 2 \mathrm{CH}$ ), 6.98 (dt, $J=15.7,6.4 \mathrm{~Hz}, 1 \mathrm{H}, \mathrm{CHCH}_{2}$ ), 5.89 (d, $J=15.7,1 \mathrm{H}, \mathrm{CHCO}$ ), $4.25\left(\mathrm{t}, J=6.4 \mathrm{~Hz}, 2 \mathrm{H}, \mathrm{OCH} \mathrm{O}_{2}\right), 3.73\left(\mathrm{~s}, 3 \mathrm{H}, \mathrm{OCH}_{3}\right), 2.39(\mathrm{~s}, 3 \mathrm{H}$, $\left.\mathrm{CH}_{3}\right), 2.39-2.33\left(\mathrm{~m}, 2 \mathrm{H}, \mathrm{CHCH}_{2}\right), 1.93-1.86\left(\mathrm{~m}, 2 \mathrm{H}, \mathrm{CH}_{2} \mathrm{CH}_{2} \mathrm{CH}_{2}\right)$. ${ }^{13} \mathrm{C}$ NMR $\left(100 \mathrm{MHz}, \mathrm{CDCl}_{3}\right): \delta 167.01(C-0), 154.30(C-0)$, 147.74 (alkenyl $C$ ), 141.54 (aromatic $C$ ), 133.18 (aromatic $C$ ), 129.53 (aromatic $C$ ), 122.01 (alkenyl $C$ ), 116.54 (aromatic $C$ ), 87.26 (alkynyl C), 80.29 (alkynyl C), $65.07\left(\mathrm{OCH}_{2}\right), 51.68$ $\left(\mathrm{OCH}_{3}\right), 29.87\left(\mathrm{CH}_{2}\right), 28.70\left(\mathrm{CH}_{2}\right), 27.07\left(\mathrm{CH}_{3}\right)$. IR (neat, $\left.\mathrm{cm}^{-1}\right)$ : 2950, 2924, 2214, 1709, 1659, 1436, 1382, 1289, 1168, 1034, 817, 747. HRMS (ESI, $m / z$ ) calcd. for $\mathrm{C}_{17} \mathrm{H}_{18} \mathrm{O}_{4} \mathrm{Na}[\mathrm{M}+\mathrm{Na}]^{+}$: 309.1103; found: 309.1102.

Methyl (E)-6-(4-methoxylphenyl)acetylenecarboxy-2-hexenoate (8c). $68 \%$ isolated yield. Oil. ${ }^{1} \mathrm{H}$ NMR $\left(400 \mathrm{MHz}, \mathrm{CDCl}_{3}\right)$ : $\delta 7.54(\mathrm{~d}, J=8.9 \mathrm{~Hz}, 2 \mathrm{H}, 2 \mathrm{CH}), 6.97(\mathrm{dt}, J=15.6,6.9 \mathrm{~Hz}, 1 \mathrm{H}$, $\mathrm{CHCH}_{2}$ ), 6.89 (d, $J=8.9 \mathrm{~Hz}, 2 \mathrm{H}, 2 \mathrm{CH}$ ), 5.88 (d, $J=15.6,1 \mathrm{H}$, $\mathrm{CHCO}), 4.24\left(\mathrm{t}, J=6.9 \mathrm{~Hz}, 2 \mathrm{H}, \mathrm{OCH}_{2}\right), 3.84\left(\mathrm{~s}, 3 \mathrm{H}, \mathrm{OCH}_{3}\right), 3.73(\mathrm{~s}$, $\left.3 \mathrm{H}, \mathrm{OCH}_{3}\right), 2.38-2.32\left(\mathrm{~m}, 2 \mathrm{H}, \mathrm{CHCH}_{2}\right), 1.92-1.85(\mathrm{~m}, 2 \mathrm{H}$, $\mathrm{CH}_{2} \mathrm{CH}_{2} \mathrm{CH}_{2}$ ). ${ }^{13} \mathrm{C}$ NMR (100 MHz, $\left.\mathrm{CDCl}_{3}\right): \delta 167.00(\mathrm{C}-0), 161.72$ (aromatic C), 154.41 (C-0), 147.76 (alkenyl $C$ ), 135.13 (aromatic $C$ ), 121.98 (alkenyl C), 114.45 (aromatic $C$ ), 111.43 (aromatic C), 87.55 (alkynyl $C$ ), 80.09 (alkynyl $C$ ), $64.98\left(\mathrm{OCH}_{2}\right)$, $55.56\left(\mathrm{OCH}_{3}\right), 51.64\left(\mathrm{OCH}_{3}\right), 28.70\left(\mathrm{CH}_{2}\right), 27.08\left(\mathrm{CH}_{2}\right)$. IR (neat, 
$\mathrm{cm}^{-1}$ ) : 2955, 2922, 2212, 1708, 1658, 1604, 1462, 1288, 1165, 1026, 835, 746. HRMS (ESI, $m / z$ ) calcd. for $\mathrm{C}_{17} \mathrm{H}_{18} \mathrm{O}_{5} \mathrm{Na}$ [M+Na]+: 325.1052; found: 325.1058.

Methyl (E)-6-(4-tert-butylphenyl)acetylenecarboxy-2-hexenoate (8d). 89\% isolated yield. Oil. ${ }^{1} \mathrm{H}$ NMR (400 $\left.\mathrm{MHz}, \mathrm{CDCl}_{3}\right)$ : $\delta 7.53(\mathrm{~d}, J=8.2 \mathrm{~Hz}, 2 \mathrm{H}, 2 \mathrm{CH}), 7.40(\mathrm{~d}, J=8.2 \mathrm{~Hz}, 2 \mathrm{H}, 2 \mathrm{CH}), 6.97$ (dt, $J=15.7,6.4 \mathrm{~Hz}, 1 \mathrm{H}, \mathrm{CHCH}_{2}$ ), 5.89 (d, $J=15.7,1 \mathrm{H}, \mathrm{CHCO}$ ), $4.25\left(\mathrm{t}, J=6.4 \mathrm{~Hz}, 2 \mathrm{H}, \mathrm{OCH}_{2}\right), 3.73\left(\mathrm{~s}, 3 \mathrm{H}, \mathrm{OCH}_{3}\right), 2.38-2.33(\mathrm{~m}$, $2 \mathrm{H}, \mathrm{CHCH}_{2}$ ), 1.93-1.86 (m, 2H, $\mathrm{CH}_{2} \mathrm{CH}_{2} \mathrm{CH}_{2}$ ), 1.32 (s, 9H, $\mathrm{CH}_{3}$ ). ${ }^{13} \mathrm{C}$ NMR (100 MHz, $\left.\mathrm{CDCl}_{3}\right): \delta 166.97(C-0), 154.54(C-0)$, 154.29 (aromatic $C$ ), 147.70 (alkenyl $C$ ), 133.04 (aromatic $C$ ), 125.77 (aromatic C), 121.98 (alkenyl C), 116.52 (aromatic $C$ ), 87.21 (alkynyl $C$ ), 80.23 (alkynyl $C$ ), $65.03\left(\mathrm{OCH}_{2}\right), 51.59$ $\left(\mathrm{OCH}_{3}\right), 35.19\left(\mathrm{C}\left(\mathrm{CH}_{3}\right)_{3}\right), 31.17\left(\mathrm{CH}_{3}\right), 28.67\left(\mathrm{CH}_{2}\right), 27.04\left(\mathrm{CH}_{2}\right)$. IR (neat, cm-1): 2960, 2219, 1716, 1658, 1436, 1290, 1201, $1171,1040,838,748$. HRMS (ESI, $m / z$ ) calcd. for $\mathrm{C}_{20} \mathrm{H}_{24} \mathrm{O}_{4} \mathrm{Na}$ $[\mathrm{M}+\mathrm{Na}]^{+}:$351.1572; found: 351.1581.

Methyl (E)-6-(4-trifluoromethyl)acetylenecarboxy-2-hexenoate (8e). 49\% isolated yield. Oil. ${ }^{1} \mathrm{H}$ NMR (400 $\mathrm{MHz}, \mathrm{CDCl}_{3}$ ): $\delta 7.70(\mathrm{~d}, J=7.7 \mathrm{~Hz}, 2 \mathrm{H}, 2 \mathrm{CH}), 7.65(\mathrm{~d}, J=7.7 \mathrm{~Hz}, 2 \mathrm{H}, 2 \mathrm{CH}), 6.97$ (dt, $J=15.5,6.9 \mathrm{~Hz}, 1 \mathrm{H}, \mathrm{CHCH}_{2}$ ), 5.89 (d, $J=15.5,1 \mathrm{H}, \mathrm{CHCO}$ ), $4.27\left(\mathrm{t}, J=6.9 \mathrm{~Hz}, 2 \mathrm{H}, \mathrm{OCH}_{2}\right), 3.73\left(\mathrm{~s}, 3 \mathrm{H}, \mathrm{OCH}_{3}\right), 2.38-2.32(\mathrm{~m}$, $\left.2 \mathrm{H}, \mathrm{CHCH}_{2}\right), 1.93-1.88\left(\mathrm{~m}, 2 \mathrm{H}, \mathrm{CH}_{2} \mathrm{CH}_{2} \mathrm{CH}_{2}\right) .{ }^{13} \mathrm{C} \mathrm{NMR}(100 \mathrm{MHz}$, $\mathrm{CDCl}_{3}$ ): $\delta 166.98(C-0), 153.67$ ( $C$-0), 147.56 (alkenyl $C$ ), 133.34 (aromatic $C$ ), 132.59 (aromatic $C$ ), 132.26 (aromatic $C$ ), 125.70 (aromatic $C$ ), $123.52\left(\mathrm{CF}_{3}\right), 122.10$ (alkenyl $C$ ), 84.42 (alkynyl C), 82.19 (alkynyl $C$ ), $65.43\left(\mathrm{OCH}_{2}\right), 51.67\left(\mathrm{OCH}_{3}\right), 28.66\left(\mathrm{CH}_{2}\right)$, $27.01\left(\mathrm{CH}_{2}\right)$. IR (neat, $\left.\mathrm{cm}^{-1}\right): 2953,2927,2230,1716,1660$, $1324,1287,1172,1067,845,747$. HRMS (ESI, $m / z$ ) calcd. for $\mathrm{C}_{17} \mathrm{H}_{15} \mathrm{O}_{4} \mathrm{NaF}_{3}[\mathrm{M}+\mathrm{Na}]^{+}:$363.0820; found: 363.0814.

Methyl (E)-7-phenylacetylenecarboxy-2-heptenoate (9a). $30 \%$ isolated yield. Oil. ${ }^{1} \mathrm{H}$ NMR (400 $\left.\mathrm{MHz} \mathrm{CDCl}_{3}\right): \delta 7.61-7.36$ (m, 5H, 5CH), $6.97\left(\mathrm{dt}, J=15.6,7.0 \mathrm{~Hz}, 1 \mathrm{H}, \mathrm{CHCH}_{2}\right), 5.86(\mathrm{~d}, J=$ 15.6, 1H, CHCO), $4.24\left(\mathrm{t}, J=6.9 \mathrm{~Hz}, 2 \mathrm{H}, \mathrm{OCH}_{2}\right), 3.73(\mathrm{~s}, 3 \mathrm{H}$, $\left.\mathrm{OCH}_{3}\right), 2.30-2.24\left(\mathrm{~m}, 2 \mathrm{H}, \mathrm{CHCH}_{2}\right), 1.79-1.56\left(\mathrm{~m}, 4 \mathrm{H}, 2 \mathrm{CH}_{2}\right) .{ }^{13} \mathrm{C}$ NMR (100 MHz, CDCl 3 ): $\delta 166.98(C-0), 154.10(C-0), 148.53$ (alkenyl $C$ ), 133.00 (aromatic $C$ ), 130.65 (aromatic $C$ ), 128.57 (aromatic C), 121.51 (alkenyl C), 119.61 (aromatic C), 86.36 (alkynyl C), 80.57 (alkynyl C), $65.59\left(\mathrm{OCH}_{2}\right), 51.43\left(\mathrm{OCH}_{3}\right)$, $31.63\left(\mathrm{CH}_{2}\right), 27.92\left(\mathrm{CH}_{2}\right), 24.37\left(\mathrm{CH}_{2}\right)$. IR (neat, $\left.\mathrm{cm}^{-1}\right): 2958$, 2924, 2201, 1722, 1701, 1660, 1460, 1259, 1024, 800, 745. HRMS (ESI, $m / z$ ) calcd. for $\mathrm{C}_{17} \mathrm{H}_{18} \mathrm{O}_{4} \mathrm{Na}[\mathrm{M}+\mathrm{Na}]^{+}$: 309.1103; found: 309.1091 .

Methyl (E)-7-(4-methylphenyl)acetylenecarboxy-2-heptenoate (9b). 49\% isolated yield. Oil. ${ }^{1} \mathrm{H}$ NMR (400 $\mathrm{MHz}, \mathrm{CDCl}_{3}$ ): $\delta 7.49(\mathrm{~d}, J=7.9 \mathrm{~Hz}, 2 \mathrm{H}, 2 \mathrm{CH}), 7.18(\mathrm{~d}, J=7.9 \mathrm{~Hz}, 2 \mathrm{H}, 2 \mathrm{CH}), 6.97$ (dt, $J=15.7,6.4 \mathrm{~Hz}, 1 \mathrm{H}, \mathrm{CHCH}_{2}$ ), 5.86 (d, $J=15.7,1 \mathrm{H}, \mathrm{CHCO}$ ), $4.24\left(\mathrm{t}, J=6.4 \mathrm{~Hz}, 2 \mathrm{H}, \mathrm{OCH}_{2}\right), 3.73\left(\mathrm{~s}, 3 \mathrm{H}, \mathrm{OCH}_{3}\right), 2.38(\mathrm{~s}, 3 \mathrm{H}$, $\left.\mathrm{CH}_{3}\right), 2.30-2.24\left(\mathrm{~m}, 2 \mathrm{H}, \mathrm{CHCH}_{2}\right), 1.78-1.56\left(\mathrm{~m}, 4 \mathrm{H}, 2 \mathrm{CH}_{2}\right) .{ }^{13} \mathrm{C}$ NMR (100 MHz, $\left.\mathrm{CDCl}_{3}\right): \delta 167.15(C-0), 154.40(C-0), 148.76$ (alkenyl $C$ ), 141.48 (aromatic $C$ ), 133.16 (aromatic $C$ ), 129.51 (aromatic C), 121.61 (alkenyl C), 116.58 (aromatic C), 87.07 (alkynyl C), 80.38 (alkynyl C), $65.68\left(\mathrm{OCH}_{2}\right), 51.63\left(\mathrm{OCH}_{3}\right)$, $31.81\left(\mathrm{CH}_{2}\right), 29.86\left(\mathrm{CH}_{2}\right), 28.08\left(\mathrm{CH}_{2}\right), 24.52\left(\mathrm{CH}_{3}\right)$. IR (neat, $\mathrm{cm}^{-1}$ ): 2957, 2925, 2217, 1708, 1658, 1435, 1382, 1290, 1169, 1038, 817, 747. HRMS (ESI, $m / z$ ) calcd. for $\mathrm{C}_{18} \mathrm{H}_{20} \mathrm{O}_{4} \mathrm{Na}$ $[\mathrm{M}+\mathrm{Na}]^{+}:$323.1259; found: 323.1247.
Methyl (E)-7-(4-methoxylphenyl)acetylenecarboxy-2-heptenoate (9c). 56\% isolated yield. Oil. ${ }^{1} \mathrm{H}$ NMR (400 $\mathrm{MHz}, \mathrm{CDCl}_{3}$ ): $\delta 7.55(\mathrm{~d}, J=8.8 \mathrm{~Hz}, 2 \mathrm{H}, 2 \mathrm{CH}), 6.97(\mathrm{dt}, J=15.6,7.0 \mathrm{~Hz}, 1 \mathrm{H}$, $\mathrm{CHCH}_{2}$ ), 6.89 (d, $J=8.8 \mathrm{~Hz}, 2 \mathrm{H}, 2 \mathrm{CH}$ ), $5.86(\mathrm{~d}, J=15.6,1 \mathrm{H}$, CHCO), 4.23 (t, J = 7.0 Hz, 2H, OCH $), 3.84\left(\mathrm{~s}, 3 \mathrm{H}, \mathrm{OCH}_{3}\right), 3.73$ (s, $\left.3 \mathrm{H}, \mathrm{OCH}_{3}\right), 2.30-2.25\left(\mathrm{~m}, 2 \mathrm{H}, \mathrm{CHCH}_{2}\right), 1.78-1.56\left(\mathrm{~m}, 4 \mathrm{H}, \mathrm{CH}_{2}\right)$. ${ }^{13} \mathrm{C}$ NMR $\left(100 \mathrm{MHz}, \mathrm{CDCl}_{3}\right): \delta 167.16(C-0), 161.68$ (aromatic C), 154.53 (C-0), 148.78 (alkenyl $C$ ), 135.12 (aromatic $C$ ), 121.61 (alkenyl $C$ ), 114.44 (aromatic $C$ ), 111.49 (aromatic $C$ ), 87.38 (alkynyl $C$ ), 80.17 (alkynyl $C$ ), $65.60\left(\mathrm{OCH}_{2}\right), 55.56$ $\left(\mathrm{OCH}_{3}\right), 51.62\left(\mathrm{OCH}_{3}\right), 31.82\left(\mathrm{CH}_{2}\right), 28.10\left(\mathrm{CH}_{2}\right), 24.53\left(\mathrm{CH}_{2}\right)$. IR (neat, $\mathrm{cm}^{-1}$ ): 2924, 2850, 2212, 1705, 1657, 1604, 1510, 1460, 1286, 1163, 1029, 835, 747. HRMS (ESI, $m / z$ ) calcd. for $\mathrm{C}_{18} \mathrm{H}_{20} \mathrm{O}_{5} \mathrm{Na}[\mathrm{M}+\mathrm{Na}]^{+}$: 339.1208; found: 339.1216.

Methyl (E)-7-(4-tert-butylphenyl)acetylenecarboxy-2-heptenoate (9d). $77 \%$ isolated yield. Oil. ${ }^{1} \mathrm{H}$ NMR (400 $\left.\mathrm{MHz}, \mathrm{CDCl}_{3}\right)$ : $\delta 7.58(\mathrm{~d}, J=8.7 \mathrm{~Hz}, 2 \mathrm{H}, 2 \mathrm{CH}$ ), 7.39 (d, $J=8.7 \mathrm{~Hz}, 2 \mathrm{H}, 2 \mathrm{CH}$ ), 6.97 (dt, $\left.J=15.6,6.4 \mathrm{~Hz}, 1 \mathrm{H}, \mathrm{CHCH}_{2}\right), 5.86(\mathrm{~d}, J=15.6,1 \mathrm{H}, \mathrm{CHCO}$ ), $4.24\left(\mathrm{t}, J=6.4 \mathrm{~Hz}, 2 \mathrm{H}, \mathrm{OCH}_{2}\right), 3.73\left(\mathrm{~s}, 3 \mathrm{H}, \mathrm{OCH}_{3}\right), 2.30-2.24(\mathrm{~m}$, $2 \mathrm{H}, \mathrm{CHCH}_{2}$ ), 1.77-1.72 (m, 2H, $\left.\mathrm{CH}_{2} \mathrm{CH}_{2} \mathrm{CH}_{2}\right), 1.63-1.56(\mathrm{~m}, 2 \mathrm{H}$, $\left.\mathrm{CH}_{2}\right) 1.32\left(\mathrm{~s}, 9 \mathrm{H}, \mathrm{CH}_{3}\right) .{ }^{13} \mathrm{C}$ NMR $\left(100 \mathrm{MHz}, \mathrm{CDCl}_{3}\right): \delta 167.08$ (C-0), 154.47 (C-0), 154.37 (aromatic C), 148.70 (alkenyl $C$ ), 133.02 (aromatic $C$ ), 125.75 (aromatic $C$ ), 121.60 (alkenyl $C$ ), 116.58 (aromatic $C$ ), 87.02 (alkynyl $C$ ), 80.33 (alkynyl $C$ ), 65.64 $\left(\mathrm{OCH}_{2}\right), 51.55\left(\mathrm{OCH}_{3}\right), 35.17\left(\mathrm{C}\left(\mathrm{CH}_{3}\right)_{3}\right), 31.77\left(\mathrm{CH}_{2}\right), 31.16$ $\left(\mathrm{CH}_{3}\right)$, $28.05\left(\mathrm{CH}_{2}\right), 24.50\left(\mathrm{CH}_{2}\right)$. IR (neat, $\left.\mathrm{cm}^{-1}\right)$ : 2962, 2216, 1716, 1660, 1436, 1290, 1201, 1170, 1034, 838, 747. HRMS (ESI, $m / z$ ) calcd. for $\mathrm{C}_{21} \mathrm{H}_{26} \mathrm{O}_{4} \mathrm{Na}[\mathrm{M}+\mathrm{Na}]^{+}$: 365.1729; found: 365.1717.

Methyl (E)-7-(4-trifluoromethyl)acetylenecarboxy-2-heptenoate (9e). 44\% isolated yield. Oil. ${ }^{1} \mathrm{H}$ NMR (400 $\mathrm{MHz}, \mathrm{CDCl}_{3}$ ): $\delta 7.71(\mathrm{~d}, J=8.2 \mathrm{~Hz}, 2 \mathrm{H}, 2 \mathrm{CH}$ ), 7.65 (d, $J=8.2 \mathrm{~Hz}, 2 \mathrm{H}, 2 \mathrm{CH}$ ), 6.97 $\left(\mathrm{dt}, J=15.5,7.0 \mathrm{~Hz}, 1 \mathrm{H}, \mathrm{CHCH}_{2}\right), 5.86(\mathrm{~d}, J=15.5,1 \mathrm{H}, \mathrm{CHCO})$, $4.26\left(\mathrm{t}, J=7.0 \mathrm{~Hz}, 2 \mathrm{H}, \mathrm{OCH}_{2}\right), 3.73\left(\mathrm{~s}, 3 \mathrm{H}, \mathrm{OCH}_{3}\right), 2.31-2.25(\mathrm{~m}$, $\left.2 \mathrm{H}, \mathrm{CHCH}_{2}\right), 1.78-1.59\left(\mathrm{~m}, 2 \mathrm{H}, 2 \mathrm{CH}_{2}\right) .{ }^{13} \mathrm{C} \mathrm{NMR}(100 \mathrm{MHz}$, $\mathrm{CDCl}_{3}$ ): $\delta 166.98$ ( $C$-0), 153.62 ( $C$-0), 148.49 (alkenyl $C$ ), 133.17 (aromatic $C$ ), 132.38 (aromatic $C$ ), 132.06 (aromatic $C$ ), 125.53 (aromatic $C$ ), $123.43\left(\mathrm{CF}_{3}\right), 121.54$ (alkenyl $C$ ), 84.10 (alkynyl C), 82.15 (alkynyl $C$ ), $65.90\left(\mathrm{OCH}_{2}\right), 51.47\left(\mathrm{OCH}_{3}\right), 31.62\left(\mathrm{CH}_{2}\right)$. $29.71\left(\mathrm{CH}_{2}\right), 27.88\left(\mathrm{CH}_{2}\right)$. IR (neat, $\left.\mathrm{cm}^{-1}\right): 2951,2228,1717$, 1658, 1324, 1288, 1174, 1067, 845, 748. HRMS (ESI, $m / z$ ) calcd. for $\mathrm{C}_{18} \mathrm{H}_{17} \mathrm{O}_{4} \mathrm{NaF}_{3}[\mathrm{M}+\mathrm{Na}]^{+}$: 377.0977; found: 377.0972 .

\section{Results and discussion}

Sequential reactions can offer efficient methods for the synthesis of a variety of different important organic compounds that would be otherwise difficult to prepare using conventional methods. Our recent studies on the carboxylative coupling [34] and olefin metathesis [56] reactions provided a platform for the synthesis of functionalized 2-alkynoates from $\mathrm{CO}_{2}$, and other simple and readily available starting materials via the combination of these two reactions. Considering the high reaction efficiency of the carboxylative coupling reaction and the use of the relatively costly Grubbs-Hoveyda catalyst in the cross-metathesis reaction, the carboxylative coupling reaction was conducted prior to the cross-metathesis reaction. 
Table 1

Ag-catalyzed carboxylative coupling of terminal alkynes, $\mathrm{CO}_{2}$, and bromides.

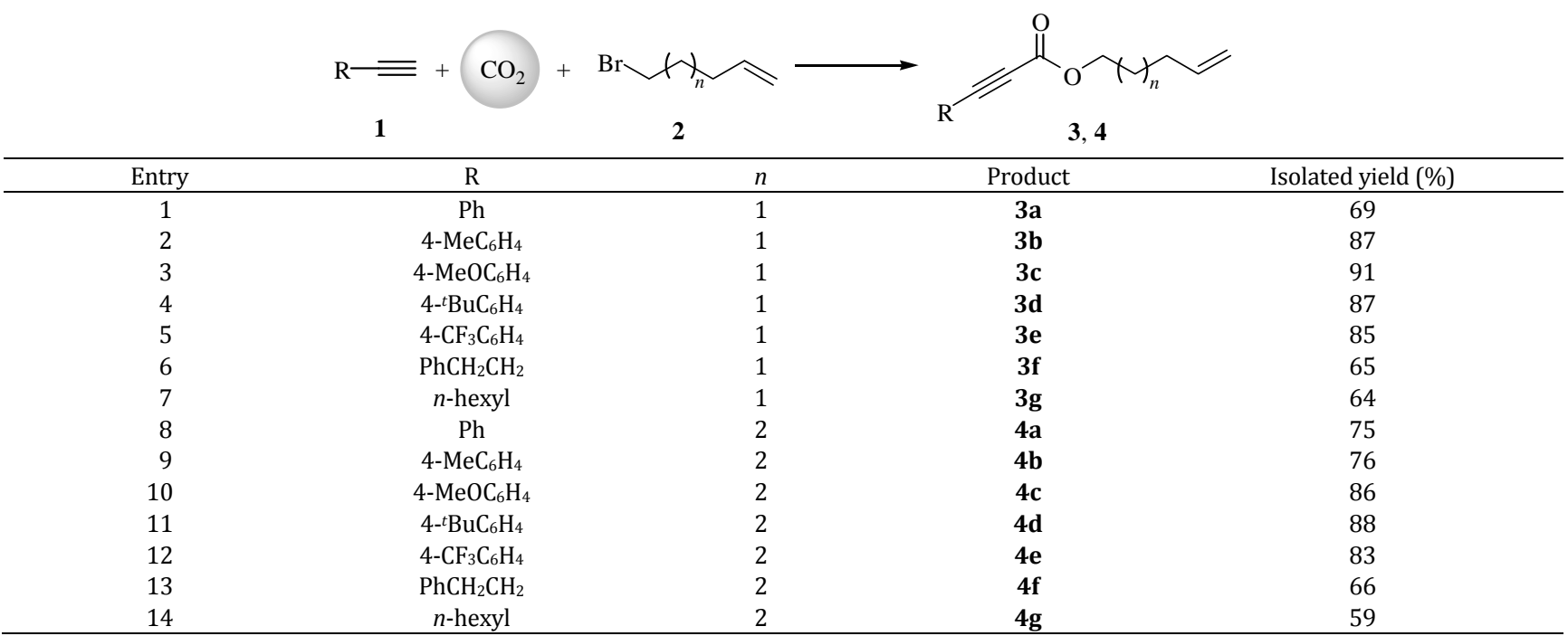

Reaction conditions: $2.0 \mathrm{mmol}$ terminal alkyne 1, $3.0 \mathrm{mmol}$ bromide $2,3.0 \mathrm{mmol} \mathrm{Cs}_{2} \mathrm{CO}_{3}, 0.002 \mathrm{mmol} \mathrm{AgI,} 2.0 \mathrm{MPa} \mathrm{CO}, 20 \mathrm{ml} \mathrm{DMF}, 60{ }^{\circ} \mathrm{C}, 24 \mathrm{~h}$.

\subsection{Ag-catalyzed carboxylative coupling of terminal alkynes, $\mathrm{CO}_{2}$, and bromides}

In the present study, the silver(I) catalytic system consisted of $0.1 \mathrm{~mol} \%$ of $\mathrm{AgI}$ and 1.5 equivalents of $\mathrm{Cs}_{2} \mathrm{CO}_{3}$, which was used as base. This catalytic system was used for the carboxylative coupling of terminal alkynes, $\mathrm{CO}_{2}$ and terminal alkene-derived bromides (5-bromopentene or 6-bromohexene) under $2.0 \mathrm{MPa} \mathrm{CO} 2$ pressure at $60{ }^{\circ} \mathrm{C}$ in DMF over a period of 24 h. As shown in Table 1, a series of 4-pentenyl 2-alkynoates (3) and 5-hexenyl 2-alkynoates (4) were obtained in moderate to good yields via the carboxylative coupling reaction of various terminal alkynes and $\mathrm{CO}_{2}$ with 5-bromopentene or 6-bromohexene, respectively. Similarly, the carboxylative reaction proceeded in a highly selective manner and no direct coupling by-products were detected even in the cases of complete conversion. Phenylacetylenes bearing both electron-donating and electron-withdrawing substituents on the ring were successfully converted to the corresponding carboxylative products in good yields (Table 1, entries 1-5 and 8-12). Compared with the aryl-substituted terminal alkynes, the alkyl-substituted terminal alkynes gave relatively low yields (Table 1 , entries $6,7,13$, and 14). This difference was attributed to the higher stability of the aryl-substituted silver(I) acetylide relative to the alkyl-substituted silver(I) acetylide intermediate which is generally thought to be initially formed according to the possible mechanism of the carboxylative coupling of the terminal alkyne, $\mathrm{CO}_{2}$, and halide [34].

\subsection{Cross-metathesis reactions of the terminal alkene-derived 2-alkynoates with methyl acrylate}

With a variety of different alkene-derived 2-alkynoates in hand, we proceeded to investigate our interest in the synthesis of functionalized 2-alkynoates via the cross-metathesis reaction of the alkene-derived 2-alkynoates with an olefin of lower reactivity. Generally, the Grubbs-Hoveyda catalyst 6 [59] shows higher catalytic activity towards cross-metathesis reactions than the second-generation Grubbs catalyst 5 [57,58] (Scheme 2). With this in mind, catalyst 6 was used in our experimental investigations. As shown in Table 2, in the presence of a 5 mol\% loading of catalyst 6 , the aryl-substituted 4-pentenyl (3a-3e) and 5-hexenyl (4a-4e) propiolates underwent cross-metathesis reactions with an excess of methyl acrylate in toluene at $60{ }^{\circ} \mathrm{C}$ to give the desired aryl-substituted methyl (E)-6-acetylenecarboxy-2-hexenoates (8a-8e) and (E)-7-acetylenecarboxy-2-heptenoates (9a-9e), respectively, in moderate to good yields (Table 2, entries 1-4, 6, and 9-13). No intermolecular or intramolecular enyne metathesis products were detected, because the electron-deficient internal alkyne moiety in the alkene-derived 2-alkynoates cannot undergo the enyne metathesis reaction with the electron-deficient olefin substrate methyl acrylate in the presence of a Grubbs-type catalyst. The second-generation Grubbs catalyst $\mathbf{5}$ showed no catalytic activity towards the cross-metathesis reaction between the alkene-derived 2-alkynoates and methyl acrylate (Table 2, entry 5). Compared with aryl-substituted 4-pentenyl propiolates $\mathbf{3 a - 3 e}$, the aryl-substituted 5-hexenyl propiolates $\mathbf{4 a}-\mathbf{4 e}$ gave relatively low yields of the cross-metathesis products. According to the well-defined olefin metathesis mechanism, the Grubbs-Hoveyda catalyst 6 initially reacted with the alkene-derived 2-alkynoates to form a new ruthenium alkyli-

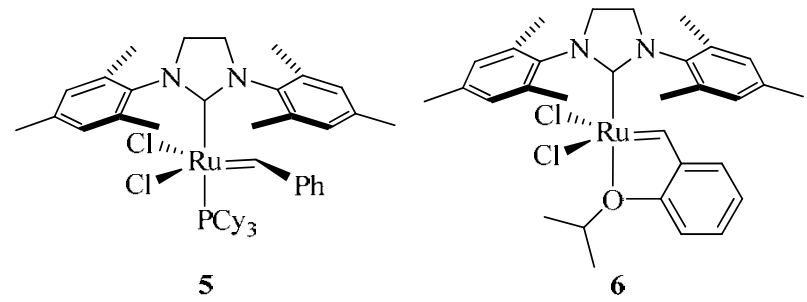

Scheme 2. The second-generation Grubbs catalyst 5 and GrubbsHoveyda catalyst 6. 
Table 2

Cross-metathesis reaction of terminal alkene-derived 2-alkynoates with methyl acrylate.

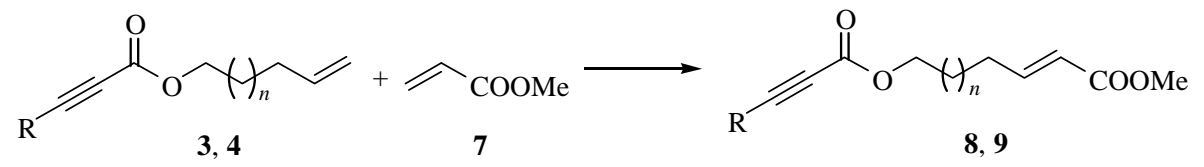

\begin{tabular}{|c|c|c|c|c|c|}
\hline \multirow{2}{*}{ Entry } & \multicolumn{3}{|c|}{3 or 4} & \multirow{2}{*}{ Product } & \multirow{2}{*}{ Isolated yield (\%) } \\
\hline & $\mathrm{R}$ & $n$ & & & \\
\hline 1 & $\mathrm{Ph}$ & 1 & $3 \mathbf{a}$ & $8 a$ & 31 \\
\hline 2 & $4-\mathrm{MeC}_{6} \mathrm{H}_{4}$ & 1 & $3 \mathbf{b}$ & $8 b$ & 63 \\
\hline 3 & $4-\mathrm{MeOC}_{6} \mathrm{H}_{4}$ & 1 & $3 c$ & $8 c$ & 68 \\
\hline 4 & ${ }^{4-}{ }^{-} \mathrm{BuC}_{6} \mathrm{H}_{4}$ & 1 & 3d & 8d & 89 \\
\hline $5^{\mathrm{a}}$ & $4-{ }^{-} \mathrm{BuC}_{6} \mathrm{H}_{4}$ & 1 & 3d & 8d & $<1^{\mathrm{c}}$ \\
\hline 6 & $4-\mathrm{CF}_{3} \mathrm{C}_{6} \mathrm{H}_{4}$ & 1 & $3 \mathbf{e}$ & $8 e$ & 49 \\
\hline 7 & $\mathrm{PhCH}_{2} \mathrm{CH}_{2}$ & 1 & $3 f$ & $8 f$ & $<1^{\mathrm{c}}$ \\
\hline 8 & $n$-hexyl & 1 & $3 g$ & $8 g$ & $<1^{\mathrm{c}}$ \\
\hline 9 & $\mathrm{Ph}$ & 2 & $4 a$ & $9 a$ & 30 \\
\hline 10 & $4-\mathrm{MeC}_{6} \mathrm{H}_{4}$ & 2 & $4 b$ & $9 b$ & 49 \\
\hline 11 & $4-\mathrm{MeOC}_{6} \mathrm{H}_{4}$ & 2 & $4 c$ & $9 c$ & 56 \\
\hline 12 & 4-t $\mathrm{BuC}_{6} \mathrm{H}_{4}$ & 2 & 4d & 9d & 77 \\
\hline 13 & $4-\mathrm{CF}_{3} \mathrm{C}_{6} \mathrm{H}_{4}$ & 2 & $4 e$ & $9 e$ & 44 \\
\hline 14 & $\mathrm{PhCH}_{2} \mathrm{CH}_{2}$ & 2 & 4f & 9f & $<1^{b}$ \\
\hline 15 & $n$-hexyl & 2 & $4 g$ & $9 g$ & $<1^{b}$ \\
\hline
\end{tabular}

Reaction conditions: $0.2 \mathrm{mmol}$ 2-alkynoates 3 or $\mathbf{4}, 10 \mathrm{mmol}$ methyl acrylate $7,0.01 \mathrm{mmol}$ Grubbs-Hoveyda catalyst $6,2 \mathrm{ml}$ toluene, $60{ }^{\circ} \mathrm{C}, 12 \mathrm{~h} .{ }^{a} 0.01$ mmol 5 as catalyst. ${ }^{b}$ Yield was determined by ${ }^{1} \mathrm{H}$ NMR.

dene complex as a catalytically active intermediate. The occurrence of chelate coordination would become possible in this newly formed complex because of the existence of carbonyl and alkyne group in 2-alkynoates substrate. Aryl-substituted 2-alkynoate substrates with longer chains gave lower yields likely because their chelate intermediates showed higher levels of stability and their catalytic activities were inhibited. Alkyl-substituted 4-pentenyl (3f and 3g) and 5-hexenyl (4f and 4g) propiolates failed to undergo the cross-metathesis reactions with methyl acrylate even at elevated temperatures, with higher catalyst loadings and with prolonged reaction times, which might be attributed to the preponderantly competitive coordination of the alkyne moiety in alkyl-substituted propiolate substrates to the ruthenium center (Table 2, entries 7, 8, 14, and 15). Other cross-metathesis partners such as acrolein, acrylonitrile and $t$-butyl acrylate gave the corresponding cross-metathesis products in very low yields $(<5 \%)$.

\section{Conclusions}

A silver-catalyzed carboxylative coupling reaction was successfully developed for the coupling of a variety of different aryl-substituted terminal alkynes and $\mathrm{CO}_{2}$ with 5-bromopentene or 6-bromohexene to give a series of 4-pentenyl 2-alkynoates or 5-hexenyl 2-alkynoates in good yields. The resulting 2-alkynoates were further transformed into methyl (E)-6-acetylenecarboxy-2-hexenoates or $(E)$-7-acetylenecarboxy-2-heptenoates in moderate to good yields via their cross-metathesis reaction with methyl acrylate in the presence of the Grubbs-Hoveyda catalyst. This carboxylative coupling/cross-metathesis reaction sequence offers an efficient and convenient method for the synthesis of functionalized 2-alkynoates from $\mathrm{CO}_{2}$, and other simple and readily available starting materials.

\section{References}

[1] Sakakura T, Choi J C, Yasuda H. Chem Rev, 2007, 107: 2365

[2] Riduan S N, Zhang Y G. Dalton Trans, 2010, 39: 3347

[3] Huang K, Sun C L, Shi Z J. Chem Soc Rev, 2011, 40: 2435

[4] Cokoja M, Bruckmeier C, Rieger B, Herrmann W A, Kuhn F. E. Angew Chem, Int Ed, 2011, 50: 8510

[5] Lü X B, Darensbourg D J. Chem Soc Rev, 2012, 41: 1462

[6] Zhang W Z, Lü X B. Chin J Catal (张文珍, 吕小兵. 催化学报), 2012, 33: 745

[7] Tsuji Y, Fujihara T. Chem Commun, 2012, 48: 9956

[8] Shi M, Nicholas K M. J Am Chem Soc, 1997, 119: 5057

[9] Yeung C S, Dong V M. JAm Chem Soc, 2008, 130: 7826

[10] Ukai K, Aoki M, Takaya J, Iwasawa N. J Am Chem Soc, 2006, 128: 8706

[11] Ohishi T, Nishiura M, Hou Z M. Angew Chem, Int Ed, 2008, 47: 5792

[12] Zhang X, Zhang W Z, Shi L L, Guo C X, Zhang L L, Lu X B. Chem Commun, 2012, 48: 6292

[13] Boogaerts I I F, Nolan S P. J Am Chem Soc, 2010, 132: 8858

[14] Zhang L, Cheng J, Ohishi T, Hou Z. Angew Chem, Int Ed, 2010, 49: 8670

[15] Mizuno H, Takaya J, Iwasawa N. J Am Chem Soc, 2011, 133: 1251

[16] Correa A, Martin R. J Am Chem Soc, 2009, 131: 15974

[17] Fujihara T, Nogi K, Xu T, Terao J, Tsuji Y. J Am Chem Soc, 2012, 134: 9106

[18] Takaya J, Iwasawa N. J Am Chem Soc, 2008, 130: 15254

[19] Williams C M, Johnson J B, Rovis T. J Am Chem Soc, 2008, 130: 14936

[20] Fujihara T, Xu T H, Semba K, Terao J, Tsuji Y. Angew Chem, Int Ed, 2011, 50: 523

[21] Li S H, Yuan W M, Ma S M. Angew Chem, Int Ed, 2011, 50: 2578

[22] Zhang L, Cheng J H, Carry B, Hou Z M. J Am Chem Soc, 2012, 134: 14314

[23] Manjolinho F, Arndt M, Goossen K, Goossen L J. ACS Catal, 2012, 2: 2014

[24] Fukue Y, Oi S, Inoue Y.J Chem Soc Chem Commun, 1994, 2091 


\title{
Graphical Abstract
}

Chin. J. Catal., 2013, 34: 1179-1186 doi: 10.1016/S1872-2067(12)60527-0

Sequential Ag-catalyzed carboxylative coupling/Ru-catalyzed cross-metathesis reactions for the synthesis of functionalized 2-alkynoates

ZHANG Linlin, ZHANG Wenzhen*, SHI Linglong, REN Xiang,

LÜ Xiaobing*

Dalian University of Technology

An efficient and convenient method has been developed for the synthesis of functionalized 2-alkynoates in good yield from terminal alkynes, $\mathrm{CO}_{2}$, terminal alkene-derived bromides, and methyl acrylate via the combination of silver-catalyzed carboxylative coupling and ruthenium-catalyzed cross-metathesis reactions.

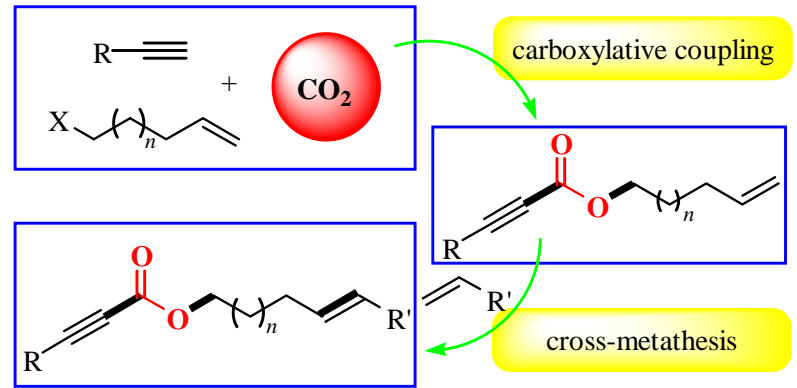

[43] Prunet J. Curr Top Med Chem, 2005, 5: 1559

[44] Donohoe T J, Bower J F, Chan L K M. Org Biomol Chem, 2012, 10: 1322

[45] Gessler S, Randl S, Blechert S. Tetrahedron Lett, 2000, 41: 9973

[46] Chatterjee A K, Choi T L, Sanders D P, Grubbs R H. J Am Chem Soc, 2003, 125: 11360

[47] Ornelas C, Mery D, Cloutet E, Aranzaes J R, Astruc D. J Am Chem Soc, 2008, 130: 1495

[48] McDougal N T, Virgil S C, Stoltz B M. Synlett, 2010, 1712

[49] Voigtritter K, Ghorai S, Lipshutz B H. J Org Chem, 2011, 76: 4697

[50] Miao X, Malacea R, Fischmeister C, Bruneau C, Dixneuf P H. Green Chem, 2011, 13: 2911

[51] Ma S, Lu X.J Org Chem, 1993, 58: 1245

[52] Rayabarapu D K, Tunge J A.J Am Chem Soc, 2005, 127: 13510

[53] Yin G, Liu G. Angew Chem, Int Ed 2008, 47: 5442

[54] Luo T, Schreiber S L. J Am Chem Soc, 2009, 131: 5667

[55] Tsujihara T, Takenaka K, Onitsuka K, Hatanaka M, Sasai H. J Am Chem Soc, 2009, 131: 3452

[56] Zhang L L, Zhang W Z, Ren X, Tan X Y, Lü X B. Tetrahedron Lett, 2012, 53: 3389

[57] Scholl M, Ding S, Lee C W, Grubbs R H. Org Lett, 1999, 1: 953

[58] Trnka T M, Morgan J P, Sanford M S, Wilhelm T E, Scholl M, Choi T L, Ding S, Day M W, Grubbs R H. J Am Chem Soc, 2003, 125: 2546

[59] Garber S B, Kingsbury J S, Gray B L, Hoveyda A H. J Am Chem Soc, 2000, 122: 8168

[41] Schrodi Y, Pederson R L. Aldrichim Acta, 2007, 40(2): 45
[42] Connon S J, Blechert S. Angew Chem, Int Ed, 2003, 42: 1900

\section{银催化羧化偶联反应及钉催化交叉复分解反应串联合成官能团炔酸酯}

\author{
张琳琳, 张文珍 ${ }^{*}$, 石凌龙, 任 祥, 吕小兵 ${ }^{*}$ \\ 大连理工大学精细化工国家重点实验室, 辽宁大连116024
}

摘要: 通过将 $\mathrm{Ag}$ 催化的羧化偶联反应与Ru催化的交叉复分解反应串联, 用端炔、二氧化碳、端烯基取代的澳代物和甲基丙烯酸 甲酯高效高产率地合成了一系列官能团化的炔酸酯.

关键词: 二氧化碳; 羧化偶联反应; 银催化剂; 交叉复分解反应; Grubbs-Hoveyda催化剂; 均相催化

收稿日期: 2012-12-07. 接受日期: 2013-01-07. 出版日期: 2013-06-20.

*通讯联系人. 电话: (0411)84986257; 传真: (0411)84986256; 电子信箱: zhangwz@dlut.edu.cn

\#通讯联系人. 电话: (0411)84986255; 传真: (0411)84986256; 电子信箱: lxb-1999@163.com

基金来源：国家自然科学基金(20802007, 21111120073); 中央高校基本科研业务费专项资金(DUT12LK47); 辽宁省教育厅科研项 目(L2012024).

本文的英文电子版由Elsevier出版社在ScienceDirect上出版(http://www.sciencedirect.com/science/journal/18722067). 